\title{
THE ORCHID CONSERVATION CHALLENGE
}

\author{
KingSLey DiXON ${ }^{1} \&$ Ryan D. PhILLIPS \\ Kings Park and Botanic Garden, West Perth, 6005 Western Australia \\ School of Plant Biology, The University of Western Australia, Nedlands, Western Australia \\ 'Author for correspondence: kdixon@bgpa.wa.gov.au
}

In the six years since the first International Orchid Conservation Congress in 2001 has the conservation of orchids progressed? Certainly orchid science has grown from less than 100 published works in the 20 year period from 1900-1920 to over 3200 for 20002005. With knowledge of orchids spanning an astonishing array of disciplines it is therefore surprising that there remains a significant gap between orchid science and orchid conservation practice. This is no more telling than in the resolutions of the plenary session of the first IOCC to adopt for orchids four targets of the 16 targets from the Global Strategy for Plant Conservation (see http://www.bgci.org/worldwide/gspc/) - that by 2010 (just three years away....!) $-90 \%$ of threatened orchids are secure in ex situ conservation collections; $50 \%$ of these threatened orchids are in active recovery programs; no orchids are threatened by unsustainable harvesting; and, every child aware of plant diversity (including orchids). How have we tracked on delivering these four targets? Besides a growth in botanic gardens to almost 2500 worldwide, the proportion of orchids in ex situ conservation collections, particularly rare and threatened taxa, has barely changed in six years yet the science and technology to achieve this target is well established. Equally the pace of the development of orchid recovery plans is outstripped by the annual increase in orchid taxa being listed as rare. Indeed the most basic of information is often lacking in orchid conservation projects from knowledge of the causal factors of orchid rarity to whether research outcomes and management plans are being converted to successes in the field? An important criterion for recovery projects should be "will it be possible to implement the results of the research - is the funding available and what are my cost-effective alternatives?' Some areas such as defining sustainability for the wild harvesting of orchids remains a complex and difficult issue often tied to local economy and cultural identity.
The slow maturation of orchids means that any wild exploitation, unless carefully managed on scientific grounds, is bound to lead to a decline in the orchid. Finally, though the final target falls outside of the expertise of conservation scientists is in the long term, it is perhaps the most critical of all conservation actions for the long term conservation of orchids. It is much easier/ preferable/more fun to do research, write and field trip than to ensure that k-12 educational needs include sound (and fun!) conservation messages. Ultimately our ability to deliver effective conservation is controlled by funding bound to political processes that in themselves rely on awareness and education from an early age.

With the long term goal of greater community awareness and funding of conservation, as researchers we can maximize our conservation outcomes in the short term through the approach we take to research and the questions we ask. When attempting to conserve a particular species, establishing which aspect of the life cycle or human interaction is driving species rarity is a critical step. A key to success in orchid reintroduction is the need for integration of knowledge gaps how many orchid reintroductions adopt a multidisciplinary approach? The majority of published works in orchid introductions rely on single principles as the basis for the reintroduction, often with a heavy emphasis on propagation/establishment techniques. However, pollination biology, mycorrhizal ecology, habitat requirements, changing habitat condition, habitat clearing and wild collecting are all potential causes of rarity that need to be considered for developing a multi-disciplinary and more sustainable conservation outcome for orchids.

An obvious division within orchid conservation biology is between the terrestrial and epiphytic life forms and the practical implications for conservation programs. For example, most terrestrial taxa have often 
intricate and biologically sensitive interactions with mycorrhiza. The evidence for such relationships in epiphytes is less convincing - take for example the billions of orchid plants produced commercially each year in the vast production houses of Europe and south-east Asia all without specific fungal endophytes. Interestingly there are far fewer epiphytic orchid reintroduction programs compared with terrestrial orchids - possibly linked to the affluence of countries (mostly temperate climates that support only terrestrial orchid floras) and their ability to support reintroduction programs.

Beyond endophyte specificity/requirements, epiphytic and terrestrial orchids share much in common in their requirements for devising an effective conservation reintroduction or preservation program. Keystone knowledge needs to reflect the ability of the reintroduced or remaining populations of the plant to complete its life cycle successfully including self-perpetuating populations. Knowledge gaps that are critical for meeting this performance indicator include: understanding pollination syndromes, biology of the pollinator, substrate, successional requirements (successional vs climax vegetation), seed germination and seedlings establishment requirements and importantly the capacity of the species and the reintroduction to cope with climate change. The latter is one of the most significant challenges facing orchid conservation programs. Evidence is continuing to mount that for terrestrial orchids, range contractions or expansions may be a fact of life for many species just as the paleoecological data indicates such changes have occurred over the millenia for other plant groups.
Given the diversity of potential drivers of orchid rarity, future conservation programs will require researchers to draw information across a wide spectrum of scientific disciplines. However, this is important in integrating conservation of orchid species with the societies in which they occur. As one of the more sensitive components of the flora to environmental change, orchids can be justified as flagship species for plant conservation, both scientifically and through their widespread recognition in the wider community.

As some of the most charismatic of species for plant conservation, failure to deliver effective conservation of orchids, even using the simple approach of the GSPC orchid targets, represents a dire scenario for conservation of all plant species. Can we as orchid conservation professionals accept the challenge of delivering a more effective orchid conservation message to the wider world? Orchids continue to be the plants that captivate and enthrall. Movies are made about them ('Adaptation' with Nic Cage and Meryl Streep), we eat them (vanilla), they remain as symbols of love and devotion and they are the most recognised of plant families. As David Attenborough stated 'Orchids surely are the most glamorous of plants...'. The challenge therefore is to conclude the third IOCC with a renewed sense of purpose and direction melding as never before, science with orchid conservation practice. And by securing orchid conservation there is a very real opportunity for collateral conservation of a considerably larger biodiversity as orchids unlike most plant groups depend for their existence upon a host of other plants and animals. What better reason for conserving these remarkable plants.

Dr Kingsley Dixon has over 20 years experience in researching the ecology and physiology of Australian native plants and ecosystems. He leads a science group comprising botanical and restoration sciences and, as Director of Science at the Botanic Gardens and Parks Authority (BGPA), has developed a strong multi-disciplinary approach to conservation and restoration of native plant biodiversity and degraded landscapes. The research team of over 40 research staff and postgraduate students specialise in seed ecology and biology, propagation science, germplasm storage, conservation genetics and restoration ecology with a strong emphasis on orchid biology and conservation. This research group has contributed significantly to seed science in Australia, with major advances in understanding seed dormancy (pioneering work in smoke technology) as well as orchid seed conservation.

Ryan Phillips is a PhD student at Kings Park and Botanic Garden and the University of Western Australia working on the role of mycorrhiza and pollinators in controlling rarity and speciation in Drakaea. Interests include the causes of orchid diversification and rarity and the co-evolution of orchids and their pollinators. 